\title{
$Q$-SETS, SIERPINSKI SETS, AND RAPID FILTERS
}

\author{
HAIM JUDAH AND SAHARON SHELAH
}

(Communicated by Andreas R. Blass)

\begin{abstract}
In this work we will prove the following:
Theorem 1. $\operatorname{cons}(Z F)$ implies $\operatorname{cons}(Z F C+$ there exists a $Q$-set of reals + there exists a set of reals of cardinality $\aleph_{1}$ which is not Lebesgue measurable).

Theorem 2. $\operatorname{cons}(Z F)$ implies cons $\left(Z F C+2^{\aleph_{0}}\right.$ is arbitrarily larger than $\aleph_{2}+$ there exists a Sierpinski set of cardinality $2^{\aleph_{0}}+$ there are no rapid filters on $\omega)$.
\end{abstract}

These theorems give answers to questions of Fleissner [Fl] and Judah [Ju].

\section{INTRODUCTION}

In this work we will solve two open problems about special sets of the reals. In order to state them we need some definitions.

0.1 . Definition. A set of reals $A$ is a $Q$-set iff every subset of $A$ is a relative $F_{\sigma}$, i.e., it is a countable union of relatively closed subsets of $A$.

$Q$-sets are very strange: for example $2^{\aleph_{0}}<2^{\aleph_{1}}$ implies that there are no $Q$-sets of cardinality $\aleph_{1}$. Also $Q$-sets have universal measure zero, but they do not necessarily have strong measure zero (see [Fl, JSh2, Mi2]).

In $[\mathrm{Fl}]$ it is asked if the existence of a $Q$-set of cardinality $\aleph_{1}$ implies that every $\aleph_{1}$-set of reals is of Lebesgue measure zero. Our first theorem answers this question negatively by showing

Theorem. $\operatorname{cons}(Z F)$ implies $\operatorname{cons}(Z F C+$ there exists a $Q$-set of reals + there exists a set of reals of cardinality $\aleph_{1}$ which is not Lebesgue measurable).

We show this theorem as follows. We begin by forcing a set $A$ of reals of cardinality $\aleph_{1}$, and then we force, with a countable support iteration of length $\omega_{2}$, making $A$ a $Q$-set in the generic extension. We prove that this composition of forcing notions satisfies the Sacks property (studied in [Sh]) and, in the end

Received by the editors December 4, 1987 and, in revised form, April 5, 1990.

1980 Mathematics Subject Classification (1985 Revision). Primary 03E35; Secondary 03E15.

The first author would like to thank the NSF for its partial support under grant DMS-8701828.

The second author thanks the United States-Israel Binational Foundation for partially supporting his research. 
of the section, we prove that if a forcing notion has the Sacks property then in the generic extension the old reals have outer measure one. Clearly this implies, if we begin from $L$, that in our generic extension there exists an uncountable $Q$-set and a $\aleph_{1}$-set of reals which is not Lebesgue measurable.

0.2. Definition. (a) A set of reals $A$ is a Sierpinski set iff for every measure zero set $M, A \cap M$ is countable.

(b) $[\omega]^{\omega}=\left\{x: x \subseteq \omega \wedge|x|=\aleph_{0}\right\} ;[\omega]^{<\omega}=\left\{x: x \subseteq \omega \wedge|x|<\aleph_{0}\right\}$.

(c) A subset $F \subseteq[\omega]^{\omega}$ is a rapid filter iff

(i) $(\forall x, y \in F)\left(x \cap y \in[\omega]^{\omega}\right)$ and $(\forall x \forall y)(x \in F \wedge x \subseteq y \rightarrow y \in F)$,

(ii) $\left(\forall f \in \omega^{\omega} \exists x \in F\right)(\forall n \in \omega)(|f(n) \cap x|<n)$.

Clearly, if the Sierpinski set has the cardinality of the continuum then the real line cannot be the union of less than $2^{\aleph_{0}}$-many measure zero sets.

In [Ju] it was remarked that if the reals are not the union of less than $2^{\aleph_{0}}$ many meager sets then there exists a rapid filter on $\omega$. Therefore it was asked: if the reals are not the union of less than $2^{\mathrm{N}_{0}}$-many measure zero sets then does there exist a rapid filter on $\omega$ ? The next theorem will answer this question negatively.

Theorem. $\operatorname{cons}(Z F)$ implies cons $\left(Z F C+2^{\aleph_{0}}\right.$ is arbitrarily larger than $\aleph_{2}+$ there exists a Sierpinski set of cardinality $2^{\aleph_{0}}+$ there are no rapid filters on $\omega)$.

This theorem has some applications. For example, the existence of a Sierpinski set of cardinality $2^{\kappa_{0}}$ implies that every $\Delta_{2}^{1}$-set of reals is measurable (see [JSh1]); also in this model $\omega_{1}^{L}=\omega_{1}$, and therefore, we get a model for "Every $\Delta_{2}^{1}$-set of reals is Lebesgue measurable $+\omega_{1}^{L}=\omega_{1}+$ there is no rapid filter on $\omega$." This says that it is impossible to improve the following result of Raisonnier [Ra]:

"If every $\Sigma_{2}^{1}$-set of reals is Lebesgue measurable and $\omega_{1}^{L}=\omega_{1}$ then there is a rapid filter on $\omega . "$

We prove this theorem in $\S 2$. The model is gotten by adding $\omega_{2}$-many Mathias reals and afterward adding random reals. It was remarked by A. Miller in [Mi1] that in the model obtained by iterating $\omega_{2}$-Mathias reals over $L$ there is no rapid filter on $\omega$.

We assume that the reader knows the material given in [Ba], about countable support iterated forcing and forcing notion satisfying the Axiom A (for the notation). The rest of the notation is standard.

\section{1. $Q$-SETS}

In this section we build a model of set theory where there exists a $Q$-set of reals and there exists an outer measure one set of reals of cardinality $\aleph_{1}$. This is the model given in 1.6. For the basic definitions the reader may consult the 
introduction $(\S 0)$ and Fleissner [Fl]. We also need some definitions used in the construction.

1.1. Definition. $\bar{A}=\left\langle a_{i}, A_{i}: i\left\langle\omega_{1}\right\rangle\right.$ is a suitable sequence if and only if

(a) $A_{i} \in[\omega]^{\omega}$ for every $i \in \omega_{1}$;

(b) if $i<j<\omega_{1}$ then $A_{i} \subseteq^{*} A_{j}\left(\exists n\left(A_{i}-n \subseteq A_{j}\right)\right)$ and $A_{j}-A_{i} \in[\omega]^{\omega}$;

(c) $a_{i} \in\left[A_{i+1}-A_{i}\right]^{\omega}$ for every $i \in \omega_{1}$.

1.2. Definition. For $\bar{A}=\left\langle a_{i}, A_{i}: i<\omega_{1}\right\rangle$ suitable, and $X \subseteq \omega_{1}$ we define the partially ordered set $P(\bar{A}, X)$ by stipulating that $h$ belongs to $P(\bar{A}, X)$ if and only if

(i) $h$ is a partial function from $\omega$ to $\{0,1\}$;

(ii) there exists $i=i(h)$ such that

$$
\text { Dom } h \subseteq^{*} A_{i} \quad \text { (take such } i \text { minimal); }
$$

(iii) for every $j<i(h)$ we have

$$
\begin{gathered}
a_{j} \subseteq^{*} \operatorname{Dom}(h), \\
\text { if } j \in X \text { then } a_{j} \subseteq^{*} h^{-1}(\{1\}), \\
\text { if } j \notin X \text { then } a_{j} \subseteq^{*} h^{-1}(\{0\}) .
\end{gathered}
$$

For $h_{1}, h_{2} \in P(\bar{A}, X)$ we set $h_{1} \leq h_{2}$ if and only if $h_{1} \subseteq h_{2}$.

1.3. Lemma. If $\bar{A}=\left\langle a_{i}, A_{i}: i<\omega_{1}\right\rangle$ and $X \subseteq \omega_{1}, P(\bar{A}, X)$ are as in 1.2 and $h \in P(\bar{A}, X)$, hence $i(h)=\alpha$ is well defined, $\alpha<\beta<\omega_{1}$, then there exists $h^{*} \in P(\bar{A}, X)$ such that

$$
h \subseteq h^{*} \text { and } i\left(h^{*}\right) \geq \beta .
$$

Proof. There exists $g:[\alpha, \beta) \rightarrow \omega$ such that

(a) $\alpha \leq \gamma<\beta$ implies $(\operatorname{Dom} h) \cap a_{\gamma} \subseteq g(\gamma) \supseteq a_{\gamma}-A_{\beta}$;

(b) $\alpha \leq \gamma<\delta<\beta$ implies $\left(a_{\gamma}-g(\gamma)\right) \cap\left(a_{\delta}-g(\delta)\right)=\varnothing$ (simply let $\left\langle\gamma_{l}: l<l^{*} \leq \omega\right\rangle=[\alpha, \beta)$ and construct $g\left(\gamma_{l}\right)$ by induction on $\left.l\right)$. Now Dom $h^{*}=(\operatorname{Dom} h) \cup \bigcup_{\gamma \in[\alpha, \beta)}\left(a_{\gamma}-g(\gamma)\right)$ and

$$
h^{*}(n)= \begin{cases}h(n) & \text { if } n \in \operatorname{Dom} h, \\ 0 & \text { if } n \in a_{\gamma}-g(\gamma) \text { and } \gamma \notin X, \\ 1 & \text { if } n \in a_{\gamma}-g(\gamma) \text { and } \gamma \in X .\end{cases}
$$

1.4. Lemma. Let $V$ be a model of $Z F C$ satisfying

(i) $\bar{A}=\left\langle a_{i}, A_{i}: i<\omega_{1}\right\rangle$ is suitable, $\bar{A} \in V$;

(ii) for every $X \subseteq \omega_{1}$ there exists $M \subseteq V$ such that $X \in M, \bar{A} \in M$, and therefore, $P(\bar{A}, X)$ is definable in $M$;

(iii) there exists $G \in V$ such that $G \subseteq P(\bar{A}, X) M$ and $G$ is generic over $M$. Then $B(\bar{A})=\left\{f \in 2^{\omega}:\left(\exists i<\omega_{1}\right)\left(\operatorname{char}\left(a_{i}\right)=f\right)\right\}$ is a $Q$-set in $V$. 
Proof. Use 1.3 and the hypothesis.

1.5. Definition. Let $\bar{Q}=\left\langle P_{i} ; Q_{j}: i<\omega_{2}, j<\omega_{2}\right\rangle$ be a countable support iterated forcing system satisfying

(a) $Q_{0}=\left\langle\left\{\left\langle a_{i}, A_{i}: i<\alpha\right\rangle: a<\omega_{1}\right.\right.$ and $\left\langle a_{i}, A_{i}: i<\alpha\right\rangle$ is an initial segment of a suitable sequence $\}, \subseteq\rangle$.

Let $\overline{\mathbf{A}}$ be the $Q_{0}$-name of the suitable sequence generated by the $Q_{0}$-generic object.

(b) Let $0<i<\omega_{2}$; then there exists a $P_{i}$-name $\mathbf{X}$ such that

$$
\Vdash_{P_{i}} \text { “X } \subseteq \omega_{1} \text { and } \mathbf{Q}_{i}=P(\overline{\mathbf{A}}, \mathbf{X}) " .
$$

(c) If $i<\omega_{2}$ and $\mathbf{X}$ is a $P_{i}$-name such that

$$
\Vdash_{P_{i}} \text { "X } \subseteq \omega_{1} "
$$

then there exists $j \geq i$ and $\mathbf{Y}$ a $P_{j}$-name satisfying

$$
\Vdash_{P_{j}} \text { “X }[\mathbf{G} \uparrow i]=\mathbf{Y} \text { and } Q_{j}=P(\overline{\mathbf{A}}, \mathbf{Y}) " \text {. }
$$

1.6. Theorem. Let $P \omega_{2}$ be the directed limit of the iterated forcing system $\bar{Q}$ defined in 1.5. Let $G \subseteq P \omega_{2}$ be generic over $V \vDash$ " $G C H$ ". Then the following holds:

(a) For every $i<\omega_{2}$

$$
\Vdash_{P_{i}} \text { “Q } Q_{i} \text { satisfies } \aleph_{2}-\text { c.c } " .
$$

Therefore $P \omega_{2}$ satisfies $\aleph_{2}-$ c.c.

(b) $\mathrm{P} \mathrm{\omega}_{2}$ is a Proper Forcing notion, moreover $\mathrm{P} \mathrm{\omega}_{2}$ satisfies the Sacks property. Therefore $V[G] \vDash " 2^{\omega} \cap V$ has outer measure one" (see 1.8).

(c) If $V[G]$ we have

$$
B(\overline{\mathbf{A}}[G]) \text { is a } Q \text {-set. }
$$

Proof. (a) easy; (c) use 1.4. The proof of (b) is sharp:

(In this work we say that a forcing notion $P$ satisfies the Sacks property iff $\left(\forall \mathbf{f} \in V^{P} \forall p \in P\right) \quad$ if $p \Vdash_{P}$ “f $\in^{\omega} V$ " then $\left(\exists q \geq p \exists g \in V \cap^{\omega} V\right)(q \Vdash$ "f $(n) \in$ $g(n) ")$ and $(\forall n \in \omega)\left(|g(n)| \leq 2^{n^{2}}\right)$.)

Let $\chi$ be sufficiently large, and $p \in P \omega_{2}$. Let $N$ be such that

$$
\begin{gathered}
N \prec\left\langle H(\chi), \varepsilon, \leq^{*}\right\rangle \quad\left(\leq^{*} \text { is some fixed well order }\right), \\
p \in N, \quad \bar{Q}, P \omega_{2} \in N, \quad\|N\|=\aleph_{0} .
\end{gathered}
$$

Set $\delta=N \cap \omega_{1}$, and let $\left\langle w_{n}: n<\omega\right\rangle$ be such that $\bigcup\left\{w_{n}: n<\omega\right\}=$ $N \cap \omega_{2}-\{0\}$

$$
w_{n} \varsubsetneqq w_{n+1}, \quad\left|w_{n}\right|=n .
$$

Also let $\left\langle\tau_{n}: n\langle\omega\rangle\right.$ be an enumeration of the $P \omega_{2}$-names of ordinal numbers that belong to $N$. Let $\left\langle\alpha_{n}: n<\omega\right\rangle$ be such that $\alpha_{n}<\alpha_{n+1}$ and $\sup _{n<\omega} \alpha_{n}=\delta$. And fix $\mathbf{h} \in N$ such that $\mathbf{h}$ is a $P \omega_{2}$-name of a function from $\omega$ to $V$. 
(*) We will choose, by induction on $\omega$, conditions $p_{n} \in P \omega_{2}$ and finite sets $u_{n}$ such that

(i) $p \leq p_{n} \leq p_{n+1} \in P \omega_{2} \cap N$;

(ii) $p_{n+1} \Vdash$ " $\tau_{n} \in N \cap$ Ord";

(iii) there exists $b_{n} \in[V]^{2^{n^{2}}}$ such that

$$
p_{n+1} \Vdash " \mathbf{h}(n) \in b_{n} " ;
$$

(iv) $u_{n} \subseteq u_{n+1} \subseteq \omega$ and $\left\|u_{n}\right\|=n$;

(v) if $\beta \in w_{m+1}-w_{m}$ and $m \leq n$ then

$$
p_{n}\left\lceil\beta \Vdash " \operatorname{Dom}\left(p_{n}(\beta)\right) \cap u_{n} \subseteq u_{m+1} " ;\right.
$$

(vi) $\alpha_{n}<\lg \left(p_{n}(0)\right)$ and $u_{n} \cap A_{\alpha_{m}} \subseteq u_{m+1}$, for $m<n$.

Claim. (*) implies (b).

Proof of the claim. Let $p_{\omega}$ be defined by

$$
p_{\omega}(0)=\left\langle a_{j}, A_{j}: i<\delta\right\rangle \cup\left\langle A_{\delta}\right\rangle
$$

when for every $n$

$$
p_{n}(0)=\left\langle a_{j}, A_{j}: i<\lg \left(p_{n}(0)\right)\right\rangle \text { and } A_{\delta}=\omega-\bigcup_{n \in \omega} u_{n} .
$$

If $\beta \in \bigcup_{n} \operatorname{Dom}\left(p_{n}\right)-\{0\}$ then, from the hypothesis, for $\left\langle p_{n}(\beta): n<\omega\right\rangle$ there exists $p_{\omega}(\beta)=\bigcup_{n} p_{n}(\beta)$ such that for every $n \in \omega p_{n}(\beta) \subseteq p_{\omega}(\beta)$. (This holds in $V^{P_{\beta}}$ where $p_{\omega}\left\lceil\beta\right.$ belongs to the generic set.) Then $p_{\omega}$ is $\left\langle N, P \omega_{2}\right\rangle$-generic and $p_{\omega} \Vdash "(\forall n)\left(\mathbf{h}(n) \in b_{n}\right) "$.

Therefore the problem is to show that the inductive construction given in $(*)$ is realizable: suppose that $p_{n}$ and $u_{n}$ were given satisfying all conditions of $(*)$.

Let $w_{n+1}=\left\{\alpha_{0}<\alpha_{1}<\cdots<\alpha_{n}<\alpha_{n+1}\right\}$. We try to extend $p_{n}$ to a condition satisfying (ii) and (iii):

Notation. If $r \in P(\overline{\mathbf{A}}, \mathbf{X})$ and $h: \omega \rightarrow\{0,1\}$ is finite then $r^{[h]} \in P(\overline{\mathbf{A}}, \mathbf{X})$ when $r^{[h]}$ is such that

$$
r^{[h]}(i)= \begin{cases}r(i) & \text { if } i \in \operatorname{Dom}(r)-\operatorname{Dom}(h), \\ h(i) & \text { if } i \in \operatorname{Dom}(h) .\end{cases}
$$

Clearly for every finite $h: \omega \rightarrow\{0,1\}$ if $r \in P(\overline{\mathbf{A}}, \mathbf{X})$ then $r^{[h]} \in P(\overline{\mathbf{A}}, \mathbf{X})$.

Let $\left\langle\left\langle h_{l}^{\alpha}: \alpha \in w_{n+1}\right\rangle: l \leq l_{0} \leq 2^{(n+1)^{2}}\right\rangle$ be a list of all $\left\langle h^{\alpha}: \alpha \in w_{n+1}\right\rangle$ such that for every $\alpha \in w_{m+1}-w_{m}$

$$
h^{\alpha}: u_{n}-u_{m+1} \rightarrow\{0,1\} .
$$

Now we choose by induction on $l \leq l_{0}, p_{n, l}$ such that

(a) $p_{n, 0}=p_{n}$. 
(b) $p_{n, l} \leq p_{n, l+1} \in P \omega_{2} \cap N$.

(c) For every $\alpha \in w_{m+1}-w_{m}, m<n$ we have

$$
p_{n, l}\left\lceil\alpha \Vdash \text { “ } \operatorname{Dom} p_{n, l}(\alpha) \cap u_{n} \subseteq u_{m+1}\right. \text { ". }
$$

(d) If $p_{n, l+1}^{\prime}$ is such that

$$
p_{n, l+1}^{\prime}(\alpha)= \begin{cases}p_{n, l+1}(\alpha)^{\left[h_{l}^{\alpha}\right]} & \text { if } \alpha \in w_{n}, \\ p_{n, l+1}(\alpha) & \text { if } \alpha \notin w_{n},\end{cases}
$$

then $p_{n, l+1}^{\prime}$ forces values for $\boldsymbol{\tau}_{i}(i \leq n+1)$ and for $\mathbf{h}(i)(i \leq n+1)$.

The induction.

for $l=0: p_{n, 0}=p_{n}$,

for $l+1: p_{n, l}$ was defined satisfying (a), (b), (c), (d), and let $p_{n, l}^{*}$ be such that

$$
p_{n, l}^{*}(\alpha)= \begin{cases}p_{n, l}(\alpha)^{\left[h_{l}^{\alpha}\right]} & \text { if } \alpha \in w_{n}, \\ p_{n, l}(\alpha) & \text { if } \alpha \notin w_{n} .\end{cases}
$$

There exists $q_{n, l} \geq p_{n, l}^{*}$ such that $q_{n, l}$ forces values for $\boldsymbol{\tau}_{i}(i \leq n+1), \mathbf{h}(i)$ $(i \leq n+1)$. Fix such $q_{n, l}$ in $N$, and then we define $p_{n, l+1}$ satisfying

$$
p_{n, l+1}(\alpha)= \begin{cases}q_{n, l}(\alpha)\left\lceil\left(\omega-\operatorname{Dom} h_{l}^{\alpha}\right)\right. & \text { if } \alpha \in w_{n}, \\ q_{n, l}(\alpha) & \text { if } \alpha \notin w_{n} .\end{cases}
$$

Clearly $p_{n, l+1}$ satisfies (a), (b), (c), and (d). $P_{n, l_{0}}$ is almost $p_{n+1}$, only we need to extend it in order to find the $u_{n+1}$ required. Clearly $p_{n, l_{0}}$ fix $2^{n^{2}}$-many possible values to $\mathbf{h}(n)$. The next lemma is exactly what we need.

1.7. Lemma. If $w \subseteq \omega_{2}$ is finite, and $p \in P \omega_{2}$ and $n<\omega$ then there exists $k \in \omega$ and $q \in P \omega_{2}$ such that

(a) $n<k<\omega$;

(b) $p \leq q$;

(c) for every $\alpha \in w$

$$
q\lceil\alpha \Vdash \text { “Dom } q(\alpha) \cap[0, n)=\operatorname{Dom} p(\alpha) \cap[0, n) ” ;
$$

(d) for every $\alpha \in w$

$$
q\lceil\alpha \Vdash “ k \notin \operatorname{Dom} q(\alpha) ” .
$$

Proof. Let $w=\left\{\alpha_{1}<\alpha_{2}<\cdots<\alpha_{m}\right\}$. We choose by (decreasing) induction

$$
q_{m}, q_{m-1}, \ldots, q_{1}, q_{0}
$$

satisfying

(i) $q_{m}=p$,

(ii) $q_{l}\left\lceil\alpha_{l} \leq q_{l-1} \in P_{\alpha_{l}}\right.$, 
(iii) $q_{l-1}$ forces less than $2^{n \times m}$-many possible values for the following names of ordinals (i.e., satisfies condition (c)):

(A) $\gamma_{l}$ such that

$$
\operatorname{Dom}\left(q_{l}\left(\alpha_{l}\right)\right)={ }^{*}\left[A_{\gamma_{l}}\right]^{q_{l-1}(0)}
$$

(remember that $Q_{0}$ fixes $A_{\gamma_{l}}$ );

(B) $k_{l}$ such that

$$
1+\max \left(\operatorname{Dom} q_{l}\left(\alpha_{l}\right)-\left[A_{\gamma_{l}}\right]^{q_{l-1}(0)}\right)<k_{l}
$$

(use the above construction in order to get such $q_{l-1}$ ).

Therefore there exists $k_{l}^{i}\left(i=1, \ldots, t_{l}<\omega\right) ; \gamma_{l}^{i}\left(i=1, \ldots, s_{l}<\omega\right)$ such that

$$
q_{l} \Vdash_{P_{\alpha_{l}}} “\left(\exists i<t_{l} \exists j<s_{l}\right)\left(\gamma_{l}=\gamma_{l}^{j} \wedge k_{l}=k_{l}^{i}\right) ” .
$$

Now let

$$
\begin{gathered}
\bar{\gamma}_{l}=\max \left\{1, \gamma_{l}^{\prime}, \ldots, \gamma_{l}^{s l}\right\}, \\
\bar{k}_{l}=\max \left\{1, k_{l}^{\prime}, \ldots, k_{l}^{t_{l}}\right\}
\end{gathered}
$$

and

$$
q=\bigcup_{l=0}^{m} q_{l}, \quad \text { i.e., if } \alpha_{0}=-1 \text { and } \alpha_{m+1}=\omega_{2}
$$

then

$$
q(\alpha)=q_{l}(\alpha) \text { where } \alpha_{l-1} \leq \alpha<\alpha_{l} .
$$

Then we choose $k$ such that

$$
k>\bar{k}_{l}(l \leq m), \quad k \notin A_{\gamma 0} \cup A_{\gamma 1} \cup \cdots \cup A_{\gamma m} .
$$

This concludes the proof of part (b) of Theorem 1.6.

1.8. Theorem. If $V$ is a model for $Z F C$ and $P \in V$ is a forcing notion satisfying the Sacks property then $\Vdash_{P} " \mu^{*}\left(2^{\omega} \cap V\right)=1$ ".

Proof. Suppose that there exists $p \in P$ such that $p \Vdash_{P}$ “ $\mu\left(2^{\omega} \cap V\right)=0$ ". Then there exists $\left(\mathbf{I}_{n}: n<\omega\right)$ such that for every $n \in \omega \mathbf{I}_{n}$ is a $P$-name of a rational interval, and

$$
\begin{gathered}
0 \Vdash \text { “ } \sum \mu\left(\mathbf{I}_{n}\right)<\infty ", \\
p \Vdash " 2^{\omega} \cap V \subseteq \bigcap_{n} \bigcup_{m \geq n} \mathbf{I}_{n} " .
\end{gathered}
$$

Then there exists $\mathbf{g}$ such that $\mathbf{g}$ is a $P$-name of a function from $\omega$ to $\omega$ and satisfying

$$
0 \Vdash \cdots \sum_{m>\mathbf{g}(n)} \mu\left(\mathbf{I}_{n}\right)<\frac{1}{2^{n^{2}+n}} ” .
$$


Fix $q \geq p$ and $g \in^{\omega} \omega$, given by the Sacks property such that

$$
q \Vdash \text { “ }(\forall n)(\mathbf{g}(n)<g(n)) ” .
$$

Again using the Sacks property, let $r \geq q$ and $\left\langle\left\langle\left\langle I_{j, i}^{k}: g(i) \leq k<g(i+1)\right\rangle\right.\right.$ : $\left.\left.j<2^{i^{2}}\right\rangle: i<\omega\right\rangle$ satisfying for every $i<\omega$, there exists $j<2^{i^{2}}$

$$
r \Vdash " \forall k \in[g(i), g(i+1))\left(\mathbf{I}_{k}=I_{j, i}^{k}\right) "
$$

and under this notation we define

$$
J_{i}=\bigcup_{j<i^{i^{2}}} \bigcup_{g(i) \leq k<g(i+1)} I_{j, i}^{k}
$$

Then for every $i$

$$
\mu\left(J_{i}\right) \leq 2^{i^{2}} \cdot \frac{1}{2^{i^{2}+i}}=\frac{1}{2^{i}} .
$$

Therefore $\sum_{i \in \omega} \mu\left(J_{i}\right)<\infty$ and this implies that there exists $x \in 2^{\omega}$ such that $x \notin \bigcap_{i} \bigcup_{j \geq i} J_{j}$, and by the hypothesis this implies that

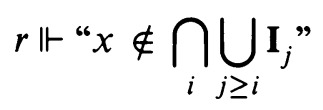

a contradiction.

It may be possible that these ideas help to solve the question in [JSh]: remember that the Sacks property implies the Laver property.

\section{RAPID FILTERS AND SIERPINSKI SETS}

2.0. Theorem. $\operatorname{cons}(Z F) \Rightarrow \operatorname{cons}\left(Z F C+\right.$ there exists a Sierpinski set $+2^{\aleph_{0}}$ is a regular cardinal + there are no rapid filters on $\omega)$.

Proof. We begin with $V=L$ and let $P$ be the $\omega_{2}$-iteration of Mathias reals with countable support and let $\mathbf{R}$ be a $P$-name of the product of $\aleph_{\alpha}$ random reals (i.e., the measure algebra). Then we will prove that if $G \subseteq P * \mathbf{R}$ then

$$
V[G] \vDash \text { "there are no rapid filters on } \omega \text { ". }
$$

Clearly this is enough in order to obtain the inclusion of the theorem. The proof of $(*)$ will take the remainder of this section.

2.1. Definition. Let a be an $R$-name of a set of ordinals; then we define

$$
\mathbf{a}(n)=\mu(\|n \in \mathbf{a}\|) \text {. }
$$

Then $\mathbf{a}()$ is a function from ordinals to $[0,1]$.

2.2. Fact. Let $M$ be a model of $Z F C$ and let $R$ be any product of random reals, i.e., a measure algebra. Let $\left\langle n_{i}: i<\omega\right\rangle$ be in $M$ an increasing sequence of natural numbers. Assume that $a$ is in $M^{R}$ and

$$
M \vDash " \Vdash \cdots(\forall k \in \omega)\left(\left|n_{k} \cap \mathbf{a}\right|<k\right) " » .
$$


Then for every $k \in \omega$

$$
M \vDash " \sum_{m=0}^{n_{k}-1} \mathbf{a}(m) \leq k " .
$$

Proof. We will work in $M$. Let $\varphi_{i}(m)$ be the formula that says

" $m$ is the $i$ th member of $a$ ".

Then $\mathbf{a}(m)=\sum_{i=0}^{m} \mu\left(\left\|\varphi_{i}(m)\right\|\right)$. Also if $m \neq n$ then $\mu\left(\left\|\varphi_{i}(m)\right\| \cdot\left\|\varphi_{i}(n)\right\|\right)=0$. Assume that there exists $k \in \omega$ such that

$$
\sum_{m=0}^{n_{k}-1} \mathbf{a}(m)>k
$$

Then

$$
\sum_{m=0}^{n_{k}-1} \sum_{i=1}^{m} \mu\left(\left\|\varphi_{i}(m)\right\|\right)>k .
$$

Therefore

$$
\left.\sum_{m=0}^{n_{k}-1} \sum_{i=1}^{k} \mu\left(\left\|\varphi_{i}(m)\right\|\right)>k \quad \text { (because } m<n_{k}\right)
$$

and hence

$$
\sum_{i=1}^{k} \sum_{m=0}^{n_{k}-1} \mu\left(\left\|\varphi_{i}(m)\right\|\right)>k
$$

And thus there exists $i$ such that $\sum_{m=0}^{n_{k}-1} \mu\left(\left\|\varphi_{i}(m)\right\|\right)>1$ and this implies that there exists $n \neq m$ such that

$$
\mu\left(\left\|\varphi_{i}(m)\right\| \cdot\left\|\varphi_{i}(n)\right\|\right) \neq \varnothing,
$$

a contradiction.

From now on we fix $M \vDash Z F C$. Let $P$ in $M$ be the $\omega_{2}$-iteration of Mathias reals. Each Mathias real adds a sequence of natural numbers. $P(0)$ is the first coordinate of $P$. Let $\left\langle\mathbf{n}_{i}: i<\omega\right\rangle$ be a $P$-name for the sequence added by $P(0)$. Let $\mathbf{R}$ be a $P$-name for a product of random reals (i.e., a measure algebra). If $H \subseteq P$ is generic over $M$ then $\mathbf{R}[H]$ is the realization of $\mathbf{R}$ in $M[H]$. Let $\mathbf{a} \in M[H]$ be an $\mathbf{R}[H]$-name for a sequence of natural numbers such that

$$
M[H] \vDash " \Vdash_{\mathbf{R}[H]} "\left|\mathbf{n}_{k}[H] \cap \mathbf{a}\right|<k ” » .
$$

Therefore, by 2.2 ,

$$
M[H] \vDash \sum_{m=0}^{n_{k}[H]-1} \mathbf{a}(m) \leq k .
$$

The function $\mathbf{a}(): \omega \rightarrow[0,1]$, defined by a, lies in $M[H]$, so it has a $P$-name. Let $\mathbf{f}$ be such a name (we omit this relation with a because it is clear). 
2.3. Lemma. Let $p \in P$, and $\varepsilon>0$ given; then there exists $w_{0} \subseteq w_{1} \subseteq \cdots \subseteq$ $w_{n} \subseteq \cdots, p_{1}^{n}, p_{2}^{n}, k_{0}<k_{1}<\cdots<k_{n}<\cdots, B_{i}^{0} \subseteq \cdots \subseteq B_{i}^{n} \subseteq \cdots, i=1,2$, satisfying

(a) for every $n, w_{n} \subseteq \omega_{2} ; k_{n} \in \omega ; p_{i}^{n} \in P, i=1,2 ; B_{i}^{n} \in[\omega]^{<\omega}$, $i=1,2 ; B_{1}^{n} \cap B_{2}^{n}=B_{1}^{0} \cap B_{2}^{0}, \bigcup B_{1}^{n} \cup \bigcup B_{2}^{n}=\omega$.

(b) $\bigcup_{n}\left\{\operatorname{Dom}\left(p_{1}^{n}\right) \cup \operatorname{Dom}\left(p_{2}^{n}\right)\right\}=\bigcup_{n} w_{n}$.

(c) For every $n, p_{i}^{n} \leq_{w_{n}}^{n} p_{i}^{n+1}, i=1,2$ (see $\left.[\mathrm{Ba}, \S 7]\right)$.

(d) $p_{i}^{0}=p$ for $i=1,2$.

(e) If $m$ is even, then for every $k>k_{m}$ there exists $q_{w_{m+1}}^{m+1} \geq p_{1}^{m}$ such that

(i) $q \Vdash_{P}$ " $\sum_{l=k_{m}}^{k} \mathbf{f}(l)<\varepsilon / 10^{m}$ ",

(ii) $q \Vdash_{P}$ “ $\sum_{\substack{l \notin B_{1}^{m} \\ l<k_{m}}} \mathbf{f}(l)<\varepsilon\left(\frac{1}{10}+\frac{1}{10^{2}}+\cdots+\frac{1}{10^{m-1}}\right)$,

(iii) $p_{2}^{m} \Vdash_{P}$ “ $\sum_{\substack{l \notin B_{2}^{m} \\ l<k_{m}}} \mathbf{f}(l)<\varepsilon\left(\frac{1}{10}+\cdots+\frac{1}{10^{m-1}}\right)$.

(f) If $m$ is odd, then for every $k>k_{m}$ there exists $q_{w_{m+1}}^{m+1} \geq p_{2}^{m}$ such that

(i) $q \Vdash$ " $\sum_{l=k_{m}}^{k} \mathbf{f}(l)<\varepsilon / 10^{m}$ ”,

(ii) $q \Vdash " \sum_{\substack{l \notin B_{2}^{m} \\ l<k_{m}}} \mathbf{f}(l)<\varepsilon\left(\frac{1}{10}+\cdots+\frac{1}{10^{m-1}}\right)$,

(iii) $p_{1}^{m} \Vdash \sum_{\substack{l \notin B_{1}^{m} \\ l<k_{m}}} \mathbf{f}(l)<\varepsilon\left(\frac{1}{10}+\cdots+\frac{1}{10^{m-1}}\right)$.

(g) $0 \in w_{0}$.

Proof. By induction on $m$.

$m=n+1$ : By the symmetry of (e) and (f) without loss of generality $n$ is even. In this stage we need to take care of the relation $\leq_{w_{n}}^{n}$.

We define $w_{n+1} \supseteq w_{n}$ such that $w_{n+1}$ contains the first $n$ numbers of each $\sup \left(p_{1}^{j}\right) \cup \sup \left(p_{2}^{j}\right)$, for $j \leq n$, in some fixed enumeration of $\sup \left(p_{1}^{j}\right) \cup \sup \left(p_{2}^{j}\right)$. that

For every natural number $\zeta$, there exists $p_{2}^{n, \zeta},\left\langle f^{\zeta, l}: l<2^{\left|w_{n+1}\right| \times n+1}\right\rangle$ such

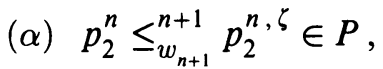

( $\beta)$ The $n+2$-th member of the infinite part of $p_{2}^{n, \zeta}(0)$ is larger than $\zeta$,

( $\gamma) \quad p_{2}^{n, \zeta} \Vdash$ “ $\exists l<2^{\left|w_{n+1}\right| \times n+1} \forall \xi<\zeta\left(\left|f^{\zeta, l}(\xi)-\mathbf{f}(\xi)\right|<2^{-t(\zeta)}\right)$ " where $t(\zeta)=$ $2^{2^{2^{\zeta+n+1+1+w_{n+1} \mid}}}$.

(Use [Ba, $\S 9.5]$ and an approximation to $\mathbf{f}(\xi)$ with error $t(\zeta)$.)

Now let $\left\langle\zeta_{i}: i<\omega\right\rangle$ be such that for every $\xi \in \omega$, and for every $l<$ $2^{\left|w_{n+1}\right| \times n+1}$ the sequence $\left\langle f^{\zeta_{i}, l}(\xi): i<\omega\right\rangle$ converges to a real number in $[0,1]$. We call this limit $f^{*, l}$. (Use a diagonal argument and the compactness theorem.)

Claim. For every $k \in \omega \quad \sum_{k<\xi<\omega} f^{*, l}(\xi) \leq n+2$. 
Proof. If not, then there exists $\bar{k}>k$ such that

$$
\sum_{k<\xi \leq k} f^{*, l}(\xi)>n+2 .
$$

Then there exists $i$ such that $\zeta_{i}>\bar{k}$ and

$$
\sum_{k<\xi \leq k} f^{\zeta_{i}, l}(\xi)>n+2
$$

and this is a contradiction to 2.2 . that

Therefore there exists $k_{n+1}>k_{n}$ such that for each $l<2^{\left|w_{n+1}\right| \times n+1}$ we have

$$
\sum_{k_{n+1}<\xi}^{\omega} f^{*}, l(\xi)<\varepsilon / 10^{n+8}
$$

Then we define

$$
\begin{aligned}
& B_{1}^{n+1}=B_{1}^{n} \cup\left[k_{n}, k_{n+1}\right), \\
& B_{2}^{n+1}=B_{2}^{n}, \\
& p_{2}^{n+1}=p_{2}^{n} .
\end{aligned}
$$

Now, by the induction hypothesis, $p_{1}^{n}$ has an extension $p_{1}^{n+1}$ such that

$$
p_{1}^{n+1} \Vdash \sum_{\substack{l \notin B_{1}^{n+1} \\ l<k_{n+1}}} \mathbf{f}(l)<\varepsilon\left(\frac{1}{10}+\cdots+\frac{1}{10^{n-1}}+\frac{1}{10^{n}}\right) .
$$

This completes the induction. The reader may check that this works.

Let $\mathbf{U}$ be a $P$-name of the $\mathbf{R}$-name for a rapid filter. Then using 2.3 we get the following

Conclusion 1. There exists $p_{1}, p_{2}, B_{1}, B_{2}$ such that

$$
\begin{aligned}
& p_{1} \Vdash_{P} \mu\left(\left\|B_{2} \in \mathbf{U}\right\|\right)<\varepsilon, \\
& p_{2} \Vdash_{P} \mu\left(\left\|B_{1} \in \mathbf{U}\right\|\right)<\varepsilon,
\end{aligned}
$$

and $B_{1} \cap B_{2}$ is finite, $B_{1} \cup B_{2}=\omega$.

Using Conclusion 1, we get the following

Conclusion 2. There exists $\delta<\omega_{2}$, such that for every $\alpha<\delta\left(\operatorname{cof}(\delta)=\omega_{1}\right)$ and for every $\left\langle B_{1}, B_{2}\right\rangle \in V\left[G\lceil\alpha]\right.$ (where $G\left\lceil\alpha\right.$ is $\mathbf{G}\left\lceil P_{\alpha}\right.$ ) if there exists $\beta$ such that in $V\left[G\lceil\beta]\right.$ Conclusion 1 holds for $B_{1}$ and $B_{2}$, then there exists $\beta<\delta$ such that in $V\left[G\lceil\beta]\right.$ Conclusion 1 holds, and $\operatorname{supp}\left(p_{1}\right) \cup \operatorname{supp}\left(p_{2}\right) \subset \delta$.

Now using Lemma 2.3, working in $V\left[G\lceil\delta]\right.$, there exists $B_{1}, B_{2}, p_{1}, p_{2}$ such that

$$
V\left[G\lceil\delta] \vDash “(*) \wedge(* *) \wedge B_{1} \cup B_{2}=\omega \wedge B_{1} \cap B_{2}\right. \text { is finite”. }
$$


(Remember that $P \cong P_{\delta \omega_{2}}$.) Then, by the hypothesis on $\delta$, and using the fact that $P_{\delta}$ is proper, we have that there exists $\beta<\delta$ and $p_{1}^{\prime}, p_{2}^{\prime}$ such that

$$
B_{i} \in V[G\lceil\beta], \quad i=1,2,
$$

and in $V\left[G\lceil\beta]\right.$ we have $(*),(* *)$ for $p_{1}^{\prime}, p_{2}^{\prime}$ respectively.

Without loss of generality $p_{1}^{\prime} \in G\left\lceil\delta\right.$. Now $p_{1}^{\prime} \cup p_{2}$ is a condition and w.l.o.g. $p_{1}^{\prime} \cup p_{2} \in G\left\lceil\omega_{2}\right.$. Therefore in $V\left[G\left\lceil\omega_{2}\right]\right.$ we have

(a) $\mu\left(\| B_{1} \in \mathbf{U}\left[G\left\lceil\omega_{2} \|\right)<\varepsilon\right.\right.$,

(b) $\mu\left(\| B_{2} \in \mathbf{U}\left[G\left\lceil\omega_{2} \|\right)<\varepsilon\right.\right.$.

If $\varepsilon$ was chosen small, we can deduce that $\mu\left(\| B_{1} \cap B_{2} \in \mathbf{U}\left[G\left[\omega_{2}\right] \|\right) \neq 0\right.$, and this implies that

$$
\mu\left(\| \mathbf{U}\left[G\left\lceil\omega_{2}\right] \text { is not a filter } \|\right)>0 \text { (because } B_{1} \cap B_{2}\right. \text { is finite) }
$$

a contradiction.

\section{REFERENCES}

[Ba] J. Baumgartner, Iterated forcing, Surveys in Set Theory (A. R. D. Mathias, ed.), London Math Soc. Lecture Notes Series 87, Cambridge Univ. Press, 1983.

[Fl] W. Fleissner, Current research on Q-sets, Topology, vol. 1, Colloq. Math. Soc. Janos Bolyai, vol. 23, North-Holland, 1980.

[Ju] H. Judah, Strong measure zero sets and rapid filters, J. Symbolic Logic 53 (1988).

[JSh1] H. Judah and S. Shelah, $\Delta \frac{1}{2}$-set of reals, Ann. Pure Appl. Logic 42.

[JSh2] _ Q Q-set do not necessarily have strong measure zero, Proc. Amer. Math. Soc. 102 (1988).

[Ku] K. Kunen, Set theory, North-Holland, 1980.

[Mil] A. Miller, There are no Q-points in Laver's model for the Borel conjecture, Proc. Amer. Math. Soc. 178 (1980).

[Mi2] _ Special subsets of the real line, Handbook of Set-Theoretic Topology (K. Kunen and J. E. Vaughan, eds.), North-Holland, 1984.

[Ra] J. Raisonnier, A mathematical proof of $S$. Shelah's theorem on the measure problem and related results, Israel J. Math. 48 (1984).

[Sh] S. Shelah, Proper forcing, Lecture Notes in Math., vol. 940, Springer-Verlag, New York.

Department of Mathematics, Bar Ilan University, 52900 Ramat Gan, IsRael

Institute of Mathematics, The Hebrew University of Jerusalem, IsRael 